\title{
Thermodynamics of Lithium Intercalation into Graphites and Disordered Carbons
}

\author{
Y. F. Reynier, R. Yazami, ${ }^{a}$ and B. Fultz ${ }^{*, z}$ \\ Division of Engineering and Applied Science, California Institute of Technology, Pasadena, \\ California 91125 USA
}

\begin{abstract}
The temperature dependence of the open-circuit potential of lithium half-cells was measured for electrodes of carbon materials having different amounts of structural disorder. The entropy of lithium intercalation, $\Delta S$, and enthalpy of intercalation, $\Delta H$, were determined over a broad range of lithium concentrations. For the disordered carbons, $\Delta S$ is small. For graphite, an initially large $\Delta S$ decreases with lithium concentration, becomes negative, and then shows two plateaus associated with the formation of intercalation compounds. For all carbons $\Delta H$ is negative, and decreases in magnitude with increased lithium concentration. For lithium concentrations less than $x=0.5$ in $\mathrm{Li}_{x} \mathrm{C}_{6}$, for the disordered carbons the magnitude of $\Delta H$ is significantly more negative than for graphite (i.e., intercalation is more exothermic). The measurements of $\Delta H$ provide an energy spectrum of chemical environments for lithium. This spectrum can be used to understand some of the concentration dependence of configurational entropy, but the negative values of $\Delta S$ require another contribution to entropy, perhaps vibrational in origin.

(c) 2004 The Electrochemical Society. [DOI: 10.1149/1.1646152] All rights reserved.
\end{abstract}

Manuscript submitted May 9, 2003; revised manuscript received September 7, 2003. Available electronically February 5, 2004.

Many methods have been used to characterize electrode materials for lithium-ion battery in the past years. In the case of the graphite anode, it is known that lithium intercalation occurs in stages, following a sequence of dilute stage 1 , stage 4 , stage 3 , liquid-type stage $2(\mathrm{~L})$, stage 2 , and stage 1 compounds. ${ }^{1}$ The stage number relates to the number of graphene layers between two lithium layers. In dilute stage 1, lithium randomly occupies the available sites between graphene layers. In liquid type stage 2 (L) compound of nominal composition $\mathrm{LiC}_{18}$, lithium atoms have no long-range ordering within the layers. This contrasts with highly ordered stage-2 $\mathrm{LiC}_{12}$ and stage- $1 \mathrm{LiC}_{6}$ compounds form a $\sqrt{2} \times \sqrt{2}$ lithium superlattice.

The temperature dependence of the open-circuit voltage $(\mathrm{OCV})$, in equilibrated half-cells can be used to determine the entropy, $\Delta S$, and enthalpy, $\Delta H$, of the lithium intercalation reaction

$$
6 \mathrm{C}+x \mathrm{Li}^{+}+x \mathrm{e}^{-} \leftrightarrow \mathrm{Li}_{x} \mathrm{C}_{6} .
$$

For an electrochemical system, the Gibbs free energy, $\Delta G$, can be related to the OCV, $E_{0} \cdot{ }^{2,3}$ In this study $\Delta G$ is defined as the OCV measured after at least $3 \mathrm{~h}$ rest

$$
\Delta G(x)=-F E_{0}(x)=\Delta H(x)-T \Delta S(x)
$$

where $F$ is the Faraday constant and $T$ the absolute temperature. The entropy of formation is

$$
\Delta S(x)=F\left(\frac{\partial E_{0}}{\partial T}\right)_{x}
$$

and enthalpy of formation is obtained from Eq. 2 and 3 as

$$
\Delta H(x)=-F E_{0}(x)+T F\left(\frac{\partial E_{0}}{\partial T}\right)_{x}
$$

In what follows, the variation of any state function, $\Delta G, \Delta H$, and $\Delta S$, relates to adding an incremental amount of lithium into the host material having lithium concentration $x$, taking metallic bcc lithium and the pure carboneous material as reference.

The OCV method has been used to study some intercalation compounds, such as $\mathrm{Li}_{x} \mathrm{TiS} 2$ or manganese oxides. ${ }^{3,4}$ However, there is only a little prior work using this method for investigating

\footnotetext{
a Permanent address: LEPMI-INPG/CNRS, 38402 St Martin d'Hères, France.

* Electrochemical Society Active Member.

z E-mail: btf@caltech.edu
}

lithium intercalation into carboneous materials, ${ }^{5-9}$ or other graphite intercalation compounds. ${ }^{10}$ Takano et al. ${ }^{11}$ studied full Li-ion batteries, which made it difficult to discuss the results. These previous investigations did not address the effect of disorder in the carbon materials, or the mechanisms of lithium intercalation. Here we report results of a temperature-dependent OCV study on how the degree of graphitization in carbon materials altered their dependence on $\Delta S$ and $\Delta H$ on lithium concentration. The effects of structural disorder on both entropy and enthalpy were large, and can be explained only in part by the configurational entropy of mixing.

\section{Experimental}

Mesocarbon microbeads (MCMB) were provided by Osaka Gas, Japan, with different heat-treatment temperatures of 750, 1000, and $2800^{\circ} \mathrm{C}$. Natural graphite from Superior Graphite, Chicago, IL, was also used for the electrochemical measurements (Ref. SO 3-24-1). The MCMB material heat-treated at $750^{\circ} \mathrm{C}$ was prepared from the so-called green powder by heating under nitrogen flow in a standard tube furnace. The temperature ramp was set to $10^{\circ} \mathrm{C}$ per hour to prevent sintering, and the final temperature was maintained for $2 \mathrm{~h}$. The two other MCMB materials were heat-treated by Osaka Gas at 1000 and $2800^{\circ} \mathrm{C}$.

Coin cells of $20 \mathrm{~mm}$ diam and $1.6 \mathrm{~mm}$ thickness were used for the OCV measurements. Half-cells containing metallic lithium as a counter electrode and the carboneous material as the working electrode were assembled in an argon-filled glove box. The electrolyte was a solution of $1 \mathrm{~m} \mathrm{LiPF}_{6}$ in an equivolumic mixture of ethyl- and dimethyl-carbonates. A microporous polyethylene separator (Tonen) was soaked overnight in the electrolyte before use.

Electrodes were made by casting a suspension in acetone of 75 wt $\%$ active material, $15 \mathrm{wt} \%$ polyvinylidene (PVDF, AtoFina, France, Ref. 2801) and $10 \mathrm{wt} \%$ acetylene black (Superior Graphite, ABG-05) into a Teflon mold, drying it overnight in air at $80^{\circ} \mathrm{C}$, and then cutting $1.27 \mathrm{~cm}$ diam samples. The electrodes, which weighed about $10 \mathrm{mg}$, were dried again overnight in vacuum at $80^{\circ} \mathrm{C}$ before admitting them into the glove box for cell assembly.

After five lithiation-delithiation cycles on an Arbin cycler between 1.5 and $0 \mathrm{~V} v s$. $\mathrm{Li}$ at a $\mathrm{C} / 5$ rate, the cells were discharged and equilibrated for over $3 \mathrm{~h}$. The OCV was monitored with a National Instrument PC-card as the temperature was decreased from room temperature to $0^{\circ} \mathrm{C}$ in several steps using a Boekel Tropicooler Peltier thermostat. Representative data are shown in Fig. 1.

The lithium concentration was deduced from the discharge time compared to the total discharge time of the fifth cycle, and the capacity bias comes from the acetylene black is corrected. Cells made with $85 \% \mathrm{ABG} 05$ and $15 \%$ PVDF had an average reversible capacity of $270 \mathrm{mAh} \mathrm{g}^{-1}$ for their fifth cycle. Based on the activation 


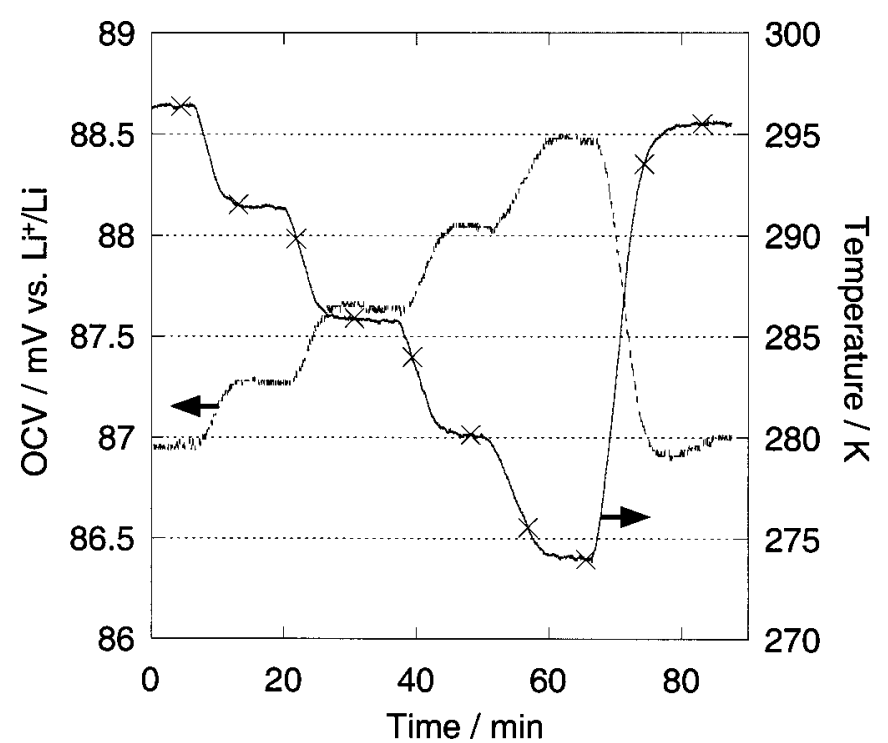

Figure 1. Evolution of the $O C V$ vs. $T$ for $\mathrm{MCMB}$ heat-treated at $2800^{\circ} \mathrm{C}$ $(x=0.67)$.

energy for self-discharge, which is $50 \mathrm{kJmol}^{-1}$ in graphite, ${ }^{12}$ a capacity loss of about $2 \%$ can be estimated for a typical ten day experiment, so that it was neglected for the composition determination.

X-ray diffraction (XRD) patterns were acquired with a Philips $\mathrm{X}$ 'pert diffractometer using monochromatic $\mathrm{Cu} \mathrm{K} \alpha$ radiation.

\section{Results}

All measurements were performed on at least two cells and showed good reproducibility. Curves of $O C V$ vs. $T$ were in good agreement with theory, as shown by the nearly perfect linear fitting in Fig. 2. The $O C V$ vs. lithium concentration curves are shown in Fig. $3 \mathrm{a}$ and $\mathrm{b}$. The experimental points were collected before each $O C V$ vs. $T$ measurement, and reflect the degree of graphitization of the compounds. For low heat-treatment temperature materials (Fig. $3 a)$, the potential slowly decreases during lithium insertion, with no

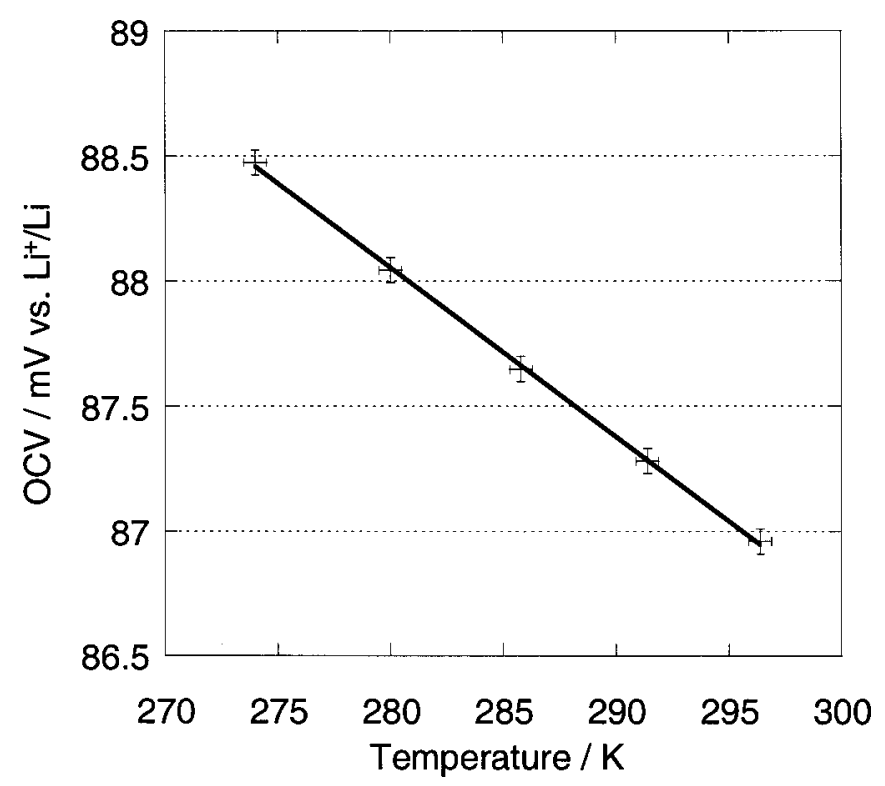

Figure 2. Fitting of the $O C V$ vs. $T$ for MCMB heat-treated at $2800^{\circ} \mathrm{C}(x$ $=0.67$ ). The linear fit gave a $\Delta S$ of $-6.5 \mathrm{Jmol}^{-1} \mathrm{~K}^{-1}$ and a $\Delta H$ of -10.3 $\mathrm{kJmol}^{-1}$ with $R=0.99974$.

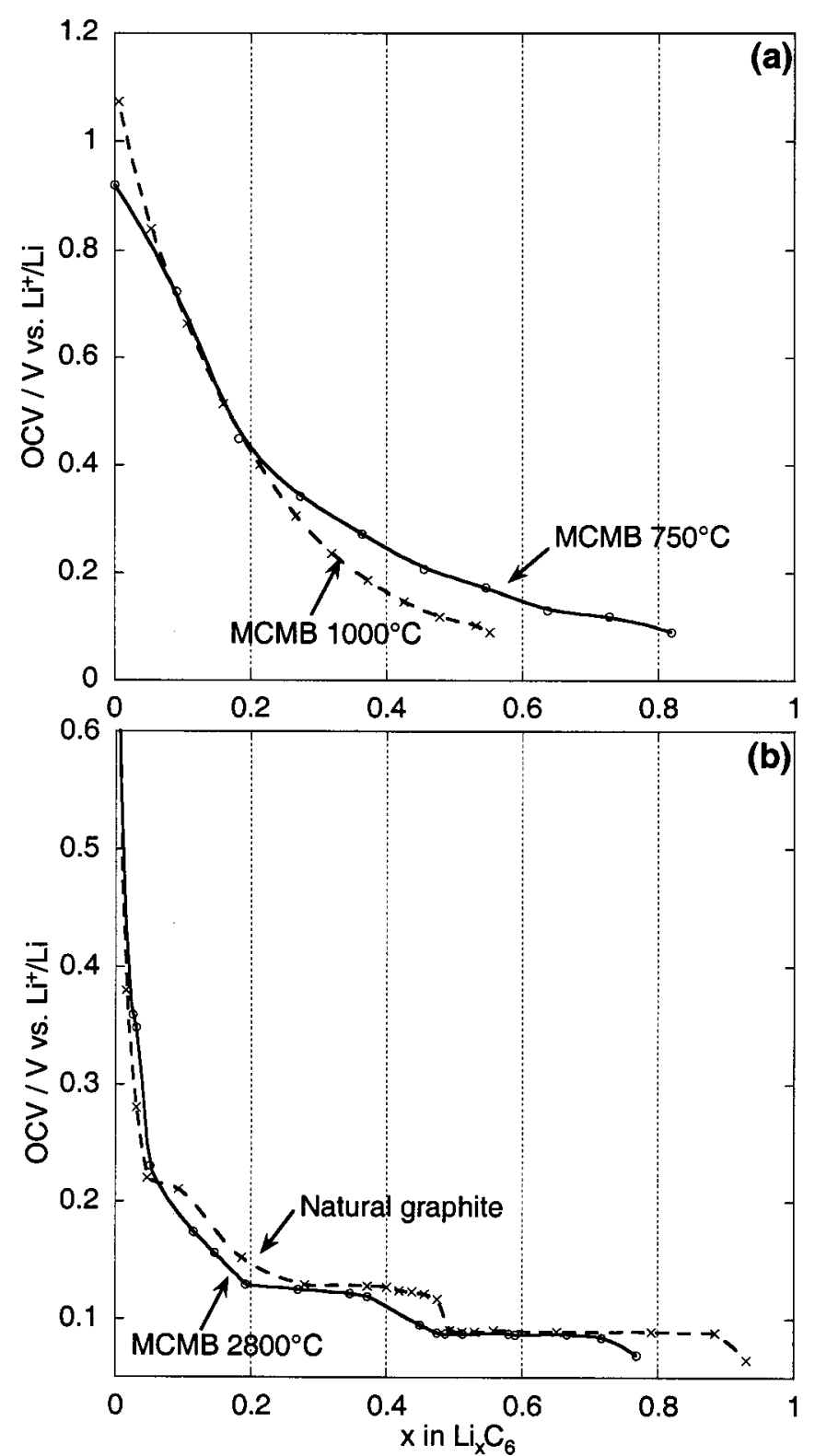

Figure 3. Evolution of the $O C V$ as a function of lithium concentration for (a) MCMB heat-treated at 750 and $1000^{\circ} \mathrm{C}$, (b) MCMB heat-treated at $2800^{\circ} \mathrm{C}$ and natural graphite.

apparent staging plateau. On the contrary, for the well-graphitized MCMB and natural graphite of Fig. 3b, characteristic plateaus appears, which denote stage formations.

Data on the entropy of intercalation, $\Delta S(x)$, are presented in Fig. $4 \mathrm{a}$ and $\mathrm{b}$. The natural graphite shows the sharpest features. After a rapid decrease, the entropy of formation levels off at about $-15 \mathrm{~J}$ $\mathrm{mol}^{-1} \mathrm{~K}^{-1}$, then increases sharply and makes a second plateau during the formation of $\mathrm{Li}_{x} \mathrm{C}_{6}$ for $x>0.5$. The MCMB graphitized at $2800^{\circ} \mathrm{C}$ shows nearly the same features, although the curve seems smoother, the initial region for dilute stage 1 compound extends further to $x \sim 0.15$, and the formation of stage 1 begins earlier at $x \sim 0.46$. These results are in good agreement with the literature. ${ }^{5,8}$ Comparatively, the two other MCMBs heat-treated at 750 and $1000^{\circ} \mathrm{C}$ show a much simpler behavior, $\Delta S(x)$ being approximately constant for all lithium concentrations. For the MCMB heat-treated at $750^{\circ} \mathrm{C}$ it is slightly negative, and slightly positive for the MCMB heat-treated at $1000^{\circ} \mathrm{C}$ 

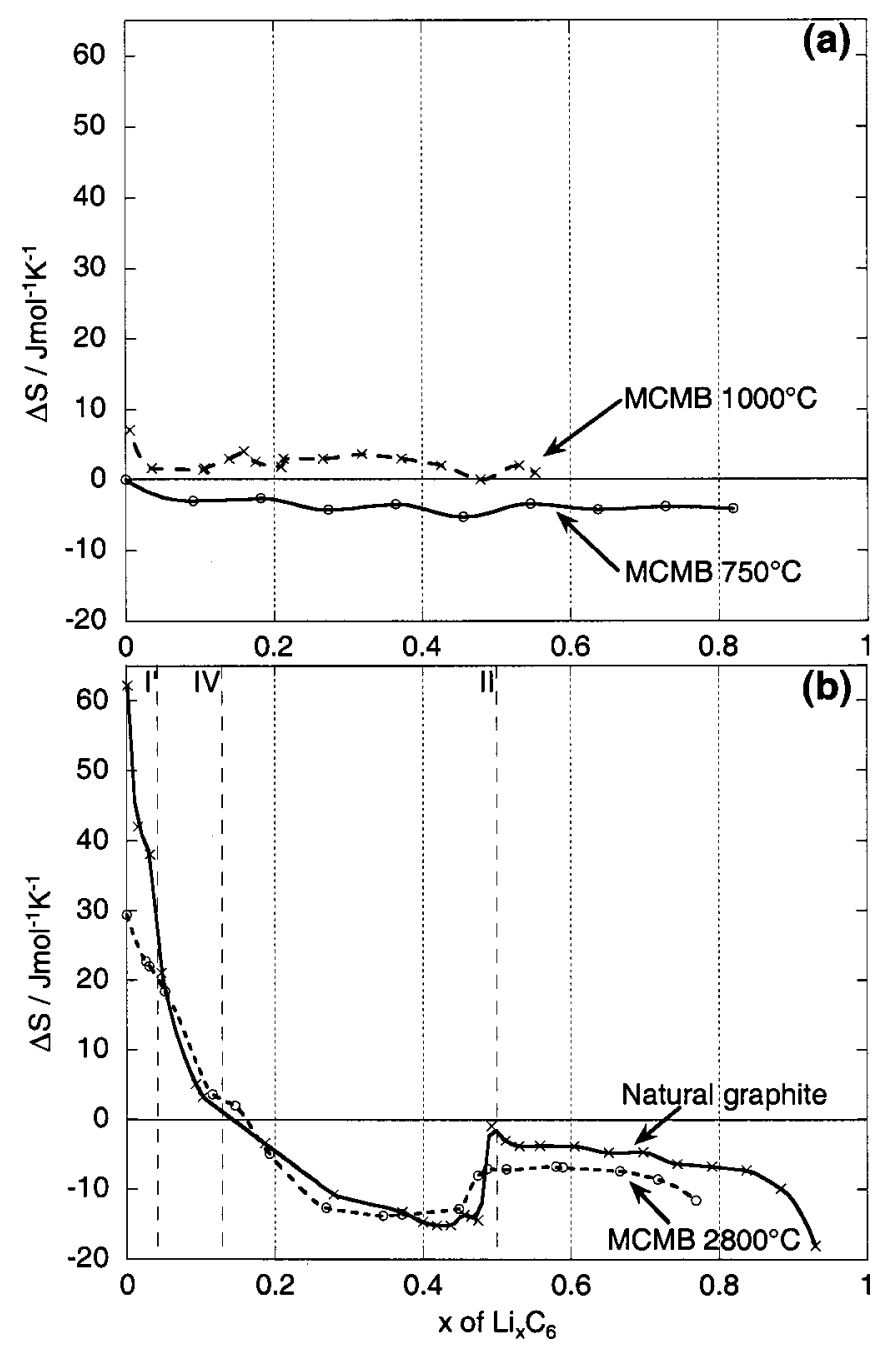

Figure 4. Evolution of $\Delta S$ as a function of lithium concentration for (a) MCMB heat-treated at 750 and $1000^{\circ} \mathrm{C}$, (b) MCMB heat-treated at $2800^{\circ} \mathrm{C}$ and natural graphite. The dash lines in (b) show stage transitions: $I^{\prime}$ $=$ dilute stage $1, \mathrm{IV}=$ stage 4 , and $\mathrm{II}=$ stage 2 .

Data on the enthalpy of intercalation, $\Delta H(x)$, are shown in Fig. 5 . The data are nearly coincident for the samples of graphite and MCMB material heat-treated at $2800^{\circ} \mathrm{C}$; the only discrepancy is seen at the end of intercalation. The capacity of the MCMB material is lower, giving a maximum lithium composition of $x=0.8$. Three enthalpy peaks can be seen at $x=0.05,0.2$, and 0.5 , corresponding to the onsets of stage 4, stage 2 , and stage 1 formation. For MCMB material heat-treated at 1000 and $750^{\circ} \mathrm{C}, \Delta H(x)$ is much larger in absolute value at low lithium concentration, and falls smoothly with no visible plateau.

\section{Discussion}

As explained in the introduction, the equilibrium OCV is the difference in chemical potential of lithium atoms in the anode and in the cathode. By making measurements at different temperatures, the enthalpy and entropy contributions to the free energy of intercalation can be independently determined as functions of the lithium concentration, as shown in Fig. 4 and 5. These changes cannot be affected by the anode of the half-cell, which is pure lithium, because all lithium atoms in the anode are in the same environments at any stage of charge. Likewise, the sizes of the carbon particles are expected to be unimportant. The chemical potential of lithium atoms depends on the local chemical environment at the atomic scale, and the fraction of surface sites is insignificant if the particles are of

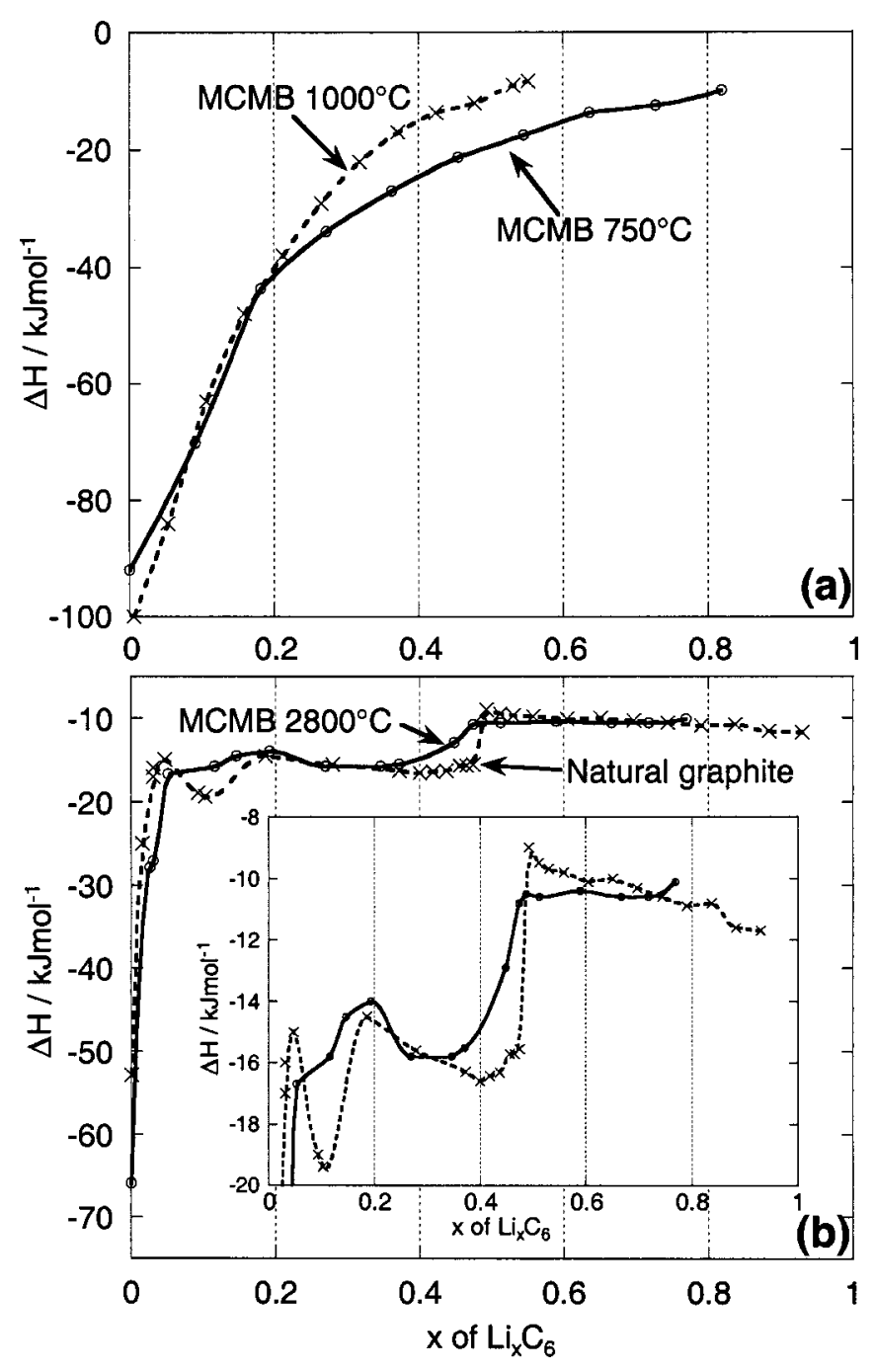

Figure 5. Evolution of $\Delta H$ as a function of lithium concentration for (a) MCMB heat-treated at 750 and $1000^{\circ} \mathrm{C}$, (b) $\mathrm{MCMB}$ heat-treated at $2800^{\circ} \mathrm{C}$ and natural graphite. Insert in (b) shows details in the $[-20 ;-10] \mathrm{kJ} \mathrm{mol}^{-1}$ range.

micrometer dimensions. For example, Thomas and Newman compared MCMB materials heat-treated at $2800^{\circ} \mathrm{C}$ with 6 and $25 \mu \mathrm{m}$ average grain sizes, and found no difference between them. ${ }^{13}$

The largest effect on the composition-dependence of the free energy of intercalation comes from the enthalpy term in the free energy. Figure 5 shows a range of the enthalpy of intercalation of nearly $100 \mathrm{~kJ} / \mathrm{mol}$, or $1 \mathrm{eV}$ per lithium atom. This greatly exceeds the range of the entropy effect, $-T S$, which is less than $23 \mathrm{~kJ} / \mathrm{mol}$ at $300 \mathrm{~K}$. Nevertheless, the entropy effects are not negligible, especially for ordered graphite at low concentrations of lithium.

The more disordered carbons show an enthalpy that increases monotonically with lithium concentration. Staging is not evident in the $O C V$ curves (Fig. 3a), nor in the enthalpy curves of Fig. 5. The range of nearly $100 \mathrm{~kJ} / \mathrm{mol}$ on the vertical axis of Fig. 5 indicates that there is a broad spectrum of chemical environments for lithium atoms in the anode. At low lithium concentrations, the insertion energy for a lithium atom differs by more than $0.5 \mathrm{eV}$ for the disordered and graphitized carbons. This presumably originates with a change in the chemical bonding to the lithium atom, perhaps worthy of investigation by electron spectroscopy. ${ }^{14} \mathrm{~A}$ wide range of energies for lithium chemical environments seems reasonable for carbons that are disordered on the atomic scale, as shown by the large breadth of the X-ray diffraction peaks in Fig. 6. From these diffrac- 


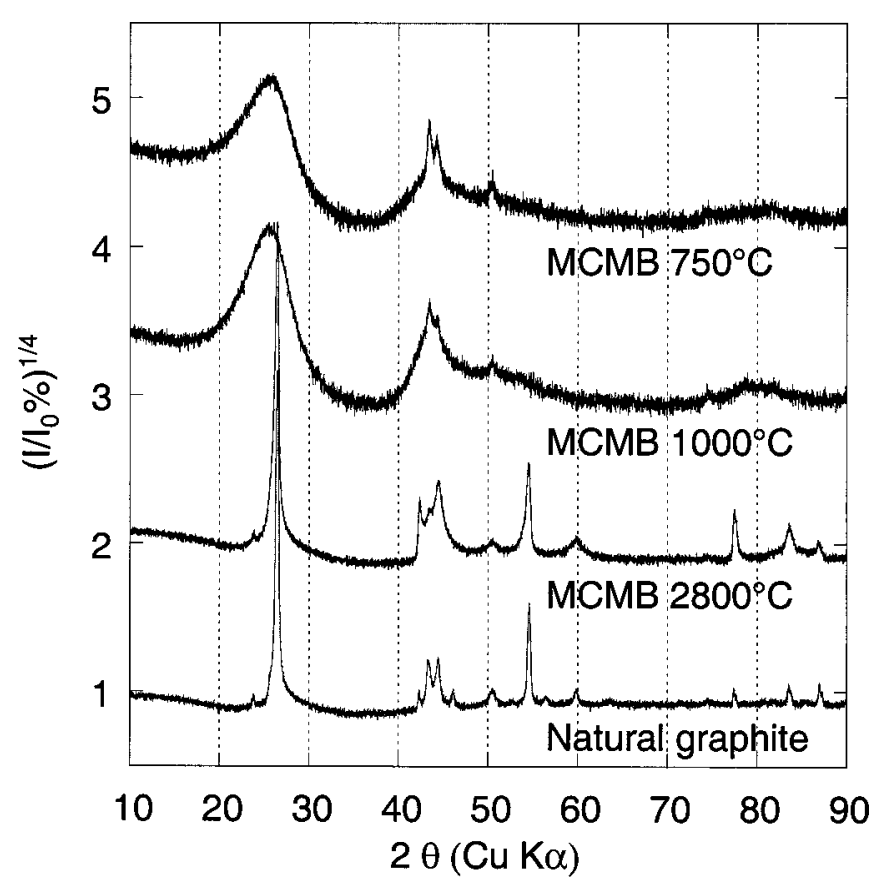

Figure 6. XRD patterns of MCMB heat-treated at $750,1000,2800^{\circ} \mathrm{C}$, and natural graphite.

tion data on the disordered MCMB materials we estimate a characteristic crystallite size $L_{\mathrm{c}}$, of about $2 \mathrm{~nm}$, whereas it exceeds $30 \mathrm{~nm}$ for graphitized carbons. Bathia et al. ${ }^{15}$ obtained similar diffraction results for $L_{\mathrm{c}}$, and also reported a $L_{\mathrm{a}}$ parameter of similar size. The crystallites in the disordered MCMB materials are less than six graphene layers thick, and have about 10 carbon hexagons along the $a$ and $b$ directions. Moreover, disordered MCMB materials are cross-linked, suppressing lithium order during intercalation.

As lithium atoms are inserted into disordered carbons at low temperatures, the most energetically favorable sites are filled first. At a finite temperature, other sites can be occupied if they are approximately within the range of $k_{\mathrm{B}} T$ from the enthalpy curve of Fig. 5 . Nevertheless, $k_{\mathrm{B}} T$ is only $2.4 \mathrm{~kJ} / \mathrm{mol}$ at $300 \mathrm{~K}$, quite small on the vertical axis of Fig. 5. The steep slopes of the enthalpy curves for disordered carbons minimize the number of sites available for lithium atoms at a particular composition. This suppresses the entropy of lithium configurations in the disordered carbons. Figure 4 shows that only the samples of MCMB heat-treated at the highest temperature and the graphite sample have large entropies of intercalation at small lithium concentrations. This is consistent with a large number of sites for lithium atoms that are nearly equivalent in energy, the enthalpy curves in Fig. 5 are essentially flat for all but perhaps the lowest concentrations of lithium. This rapid drop of $\Delta S$ in graphitized carbons can be well explained with entropy of mixing during the formation of dilute stage 1 graphite-lithium, given by Eq. 5

$$
S_{\text {config }}(x)=-k_{\mathrm{B}}[x \ln x+(1-x) \ln (1-x)]
$$

The entropy of formation per lithium mole then can be written for a disordered phase ranging from zero to $x_{0}$

$$
\Delta S(x)=R \ln \left(\frac{x_{0}-x}{x}\right)
$$

A fit of Eq. 6 to the experimental data of the natural graphite (Fig. 4) gave $x_{0} \sim 0.3$.

Although a large configurational entropy of intercalation requires a large number of equivalent sites for lithium atoms, a flat curve for enthalpy of intercalation is not a sufficient condition for a high en- tropy of intercalation. A large entropy of intercalation is not found for graphite with a concentration in the range $0.5<x<1.0$ (Fig. 4). In this range of composition, it is known that the stage 2 compound of composition, $\mathrm{LiC}_{12}$, is transforming into the stage 1 compound of composition, $\mathrm{LiC}_{6}$. The transformation proceeds by the nucleation and growth of stage I compound, with a distinct interface between the two phases. Both phases are approximately stoichiometric. The only configurational disorder is perhaps associated with the many equivalent ways of making an interface between the two phases, but this is likely a contribution only when a little stage 1 compound has formed and its surface/volume ratio is large. Perhaps the uptick in the entropy curve of the graphite compound in a small composition range just above $x=0.5$ is caused by interfacial entropy. Approximately, however, the entropy of intercalation is unchanged as the ordered stage 1 compound forms in the composition range $0.5<x<1.0$.

Over broad ranges in composition, Fig. 4 shows that the entropy of intercalation is negative for several samples. As we discussed previously, ${ }^{6}$ this is not likely to originate with configurational entropy. The configurational entropy of the lithium metal anode is zero, and the configurational entropy of a fully ordered stage 1 or stage 2 compound is also zero. This total configurational entropy is zero, but the change in configurational entropy upon intercalation of additional lithium cannot be negative. It is possible that the configurational entropy in the initial stage 1 or stage 2 compounds is positive, but the entropy curves of graphite in Fig. 4 are essentially flat when they are negative. These flat curves are typical of nucleating and growing a phase with constant thermodynamic properties. The negative entropy of lithium intercalation into graphite was attributed to the reduced vibrational entropy of lithium atoms when inserted between graphene layers, since the magnitude of the effect is approximately consistent with the vibrational frequency information measured previously by inelastic neutron scattering. ${ }^{6}$

It is probably not possible to associate the small entropies of the MCMB samples of disordered carbons with specific sources. Both the vibrational entropy and the configurational entropy are altered by atomic-scale disorder, so separation of these effects is not simple. We note, however, that the entropies of intercalation depend markedly on the state of order in the carbon. Perhaps the entropy of intercalation can be useful as a low-cost fingerprint of the state of order in carbon electrode materials, but this may require further study.

\section{Conclusions}

Measurements of the equilibrated OCV vs. temperature were used to determine the entropy and enthalpy of lithiation for carbon materials having different structural disorder. For the more disordered carbons, the entropy of lithiation was small and almost independent of lithium concentration, whereas for well-graphitized materials it showed sharper features at stage transitions. The enthalpy of lithiation showed that disordered carbons have many sites for lithium atoms of lower energy than for the graphitized samples. In graphitized compounds the enthalpy follows the staging process, showing peaks when a new phase appears. The enthalpy measurements showed a large number of energetically similar sites for lithium in dilute graphite material. These result in a large configurational entropy of intercalation. Upon further lithiation, ordered stage 2 and 1 compounds form, and the entropy of intercalation is negative, indicating that another source of entropy is involved, probably vibrational in origin.

\section{Acknowledgments}

This work was supported by DOE through Basic Energy Sciences Grant DE-FG03-00ER15035.

California Institute of Technology assisted in meeting the publication costs of this article. 


\section{References}

1. R. Yazami, Ph.D. thesis, Grenoble University Grenoble, France (1985).

2. A. J. Bard and L. R. Faulkner, Electrochemical Methods, Fundamentals, and Applications, 2nd ed., p. 48, John Wiley, New York (2000).

3. J. R. Dahn and R. R. Hearing, Can. J. Phys., 1093, 61 (1983).

4. K. E. Thomas, C. Bogatu, and J. Newman, J. Electrochem. Soc., 148, A570 (2001).

5. K. E. Thomas and J. Newman, J. Power Sources, 844, 119 (2003).

6. Y. Reynier, R. Yazami, and B. Fultz, J. Power Sources, 850, 119 (2003).

7. S. Al Hallaj, R. Venkatachalapathy, J. Prakash, and J. R. Selman, J. Electrochem. Soc., 147, 2432 (2000).

8. W. Lu and J. Prakash, J. Electrochem. Soc., 150, A262 (2003).

9. R. Yazami, in Lithium Batteries New Materials, Developments and Perspectives,
Vol. 5, C. Pistoia, Editor, p. 80, Elsevier, Amsterdam (1994).

10. R. Yazami, in Chemical Physics of Intercalation Vol. 172, A. P. Legrand and S. Flandrois, Editors, p. 464, NATO ASI Series B (1987).

11. K. Takano, Y. Saito, K. Kanari, K. Nozaki, K. Kato, A. Negishi, and T. Kato, J. Appl. Electrochem., 32, 251 (2002).

12. Y. Reynier, R. Yazami, and B. Fultz, in Proceedings of IEEE 02TH8576, p. 145 Long Beach, CA (2002).

13. K. E. Thomas and J. Newman, Abstract 346, Proceedings of the International Meeting on Lithium Batteries, IMLB'11, Monterey, CA, June 23-28, 2002.

14. A. Hightower, C. C. Ahn, P. Rez, and B. Fultz, Appl. Phys. Lett., 77, 237 (2000).

15. G. Bathia, R. K. Aggarwal, N. Punjabi, and O. P. Bahl, J. Mater. Sci., 32, 135 (1997). 